\title{
TORSION OF A CIRCULAR CYLINDER HAVING A SPHERICAL CAVITY*
}

\author{
BY \\ CHIH-BING LING \\ Aeronautical Research Laboratory, Taiwan, China
}

Abstract. This paper presents a torsion solution, based on the Michell-Föppl theory, for an infinite circular cylinder having a symmetrically-located spherical cavity. Numerical values are also given to show, in particular, the effect of the cavity on the maximum shear stress in the cylinder.

The general theory. Let $(r, \theta, z)$ be the cylindrical coordinates of a point. For convenience, $r$ and $z$ will be considered as dimensionless quantities each measured by a unit of certain typical length $a$.

The solution of the torsion problem for a solid of revolution, requires a function $\psi$ which satisfies the following differential equation. ${ }^{1}$

$$
\frac{\partial^{2} \psi}{\partial r^{2}}-\frac{3}{r} \frac{\partial \psi}{\partial r}+\frac{\partial^{2} \psi}{\partial z^{2}}=0
$$

in which the axis of revolution is taken as the $z$ axis. The two non-vanishing stress components are expressed by

$$
\tau_{\theta z}=\frac{\mu}{r^{2}} \frac{\partial \psi}{\partial r}, \quad \tau_{r \theta}=-\frac{\mu}{r^{2}} \frac{\partial \psi}{\partial z},
$$

where $\mu$ is the modulus of rigidity. The condition that the surface is free from traction takes the form

$$
\psi=\text { const. }
$$

on the entire surface.

The constant for $\psi$ at the internal surface will henceforth be taken as zero as no generality is lost in doing so. The terminal couple acting on the cylinder is then equal to

$$
T=2 \pi \mu a^{3} \psi_{e},
$$

where $\psi_{e}$ is the value of $\psi$ at the external surface.

The above theory is due to Michell and was rediscovered by Föppl.

Solutions in cylindrical and spherical coordinates. If we put

$$
\psi=r^{2} \Psi
$$

where $\Psi$ is a function of $r$ and $z$, the preceding differential equation becomes

$$
\frac{\partial^{2} \Psi}{\partial r^{2}}+\frac{1}{r} \frac{\partial \Psi}{\partial r}-\frac{4 \Psi}{r^{2}}+\frac{\partial^{2} \Psi}{\partial z^{2}}=0 .
$$

Let us assume a normal solution of $\Psi$ in the form

$$
\Psi=F(r) \stackrel{\sin k z}{\cos k z} .
$$

*Received April 11, 1951.

iSee A. E. H. Love, Mathematical theory of elasticity, 4th edition. Dover Publications, New York, 1944, pp. 325-326. 
It then appears that the function $F$ satisfies Bessel's equation

$$
\frac{d^{2} F}{d r^{2}}+\frac{1}{r} \frac{d F}{d r}-\left(k^{2}+\frac{4}{r^{2}}\right) F=0 .
$$

This equation is satisfied by $I_{2}(k r)$ and $K_{2}(k r)$ which are modified Bessel functions of order two, of the first and second kinds, respectively.

The above solution holds for any value of $k$. A more general solution of $\psi$ is therefore expressed by the following four integrals:

$$
\psi=r^{2} \int_{0}^{\infty} f(k){ }_{K_{2}(k r)}^{I_{2}(k r)} \times \cos k z^{\sin k z} d k,
$$

where in each integral $f$ is an arbitrary function of $k$.

Now we proceed to find the solution in the spherical coordinates $(\rho, \phi, \theta)$ which are connected with the cylindrical coordinates by

$$
z=\rho \cos \phi, \quad r=\rho \sin \phi, \quad \theta=\theta .
$$

Consequently, the differential operators are transformed to

$$
\begin{aligned}
& \frac{\partial}{\partial z}=\cos \phi \frac{\partial}{\partial \rho}-\frac{\sin \phi}{\rho} \frac{\partial}{\partial \phi}, \\
& \frac{\partial}{\partial r}=\sin \phi \frac{\partial}{\partial \rho}+\frac{\cos \phi}{\rho} \frac{\partial}{\partial \phi} .
\end{aligned}
$$

The differential equation for $\Psi$ becomes

$$
\frac{\partial^{2} \Psi}{\partial \rho^{2}}+\frac{2}{\rho} \frac{\partial \Psi}{\partial \rho}+\frac{1}{\rho^{2}} \frac{\partial^{2} \Psi}{\partial \phi^{2}}+\frac{\cot \phi}{\rho^{2}} \frac{\partial \Psi}{\partial \phi}-\frac{4 \Psi}{\rho^{2} \sin ^{2} \phi}=0
$$

Assume a normal solution in the form

$$
\Psi=G(\phi) \rho^{n} .
$$

It follows that the function $G$ satisfies the following equation

$$
\frac{d^{2} G}{d \phi^{2}}+\cot \phi \frac{d G}{d \phi}+\left\{n(n+1)-\frac{4}{\sin ^{2}} \frac{\phi}{\phi}\right\} G=0 .
$$

This equation is known as Legendre's associated equation and is satisfied by $P_{n}^{2}(\cos \phi)$ and $Q_{n}^{2}(\cos \phi)$, which are Legendre's associated functions of degree $n$ and order two, of the first and second kinds, respectively.

It is noted that the same Legendre's associated equation is obtained if we assume instead

$$
\Psi=\frac{G(\phi)}{\rho^{n+1}} .
$$

Since the preceding solution is valid for any integral value of $n$ including zero, a more general solution of $\psi$ is expressed by the following four series:

$$
\psi=r^{2} \sum_{n=0}^{\infty} A_{n} P_{n}^{2}(\cos \phi) \times \rho^{n}(\cos \phi) \times \rho^{-n-1},
$$

where $A_{n}$ are parametric coefficients. Note that $P_{n}^{2}$ vanishes when $n=0$ or 1 . 
Method of solution. Now, consider an infinite circular cylinder of radius $a$ (i.e., $r=1$ ) having a symmetrically-located spherical cavity of radius $\lambda a$ (i.e., $\rho=\lambda$ ), which is under torsion by terminal couples about the axis of the cylinder. For convenience, the centre of the cavity will be placed at the origin.

The method of solution is to assume $\psi$ as being composed of two parts as follows:

$$
\psi=\psi_{0}+\psi_{1},
$$

where $\psi_{0}$ is the solution of a corresponding cylinder without a cavity, while $\psi_{1}$ is an auxiliary solution which vanishes on the surface of the cylinder. The latter is added to $\psi_{0}$ so that the remaining boundary condition on the surface of the cavity is satisfied by adjusting the parametric coefficients involved in the function. The function $\psi_{0}$ is

$$
\psi_{0}=\frac{1}{4} \tau a r^{4},
$$

where $\tau$ is a constant. Since $\psi_{1}$ vanishes on the surface of the cylinder, it follows that the terminal couple is equal to $\frac{1}{2} \pi \mu \tau a^{4}$.

The auxiliary function $\psi_{1}$ may be constructed by combining linearly a set of functions each of which satisfies the given differential equation and vanishes on the surface of the cylinder. It appears that the set of functions can be derived from the following particular solution for $\psi$, namely:

$$
\psi^{*}=\frac{r^{2}}{\rho^{3}} P_{2}^{2}(\cos \phi)+r^{2} \int_{0}^{\infty} f(k) I_{2}(k r) \cos k z d k .
$$

It is obvious that the function so constructed satisfies the given differential equation. Note that this function is even in $z$ or $\cos \phi$ and, besides, it has a singularity at the origin.

According to the definition by Hobson, ${ }^{2} P_{2}^{2}(\cos \phi)$ is equal to $3 \sin ^{2} \phi$. Hence $\psi^{*}$ vanishes when $r=1$ provided that

$$
\int_{0}^{\infty} f(k) I_{2}(k) \cos k z d k=-\frac{3}{\left(1+z^{2}\right)^{5 / 2}} .
$$

A Fourier transform gives

$$
f(k)=-\frac{2}{\pi} \frac{3}{I_{2}(k)} \int_{0}^{\infty} \frac{\cos k z d z}{\left(1+z^{2}\right)^{5 / 2}}=-\frac{2 k^{2} K_{2}(k)}{\pi I_{2}(k)}
$$

The last result is a particular case of the integral considered by Poisson and Malmstén. ${ }^{3}$ The function $\psi^{*}$ is thus fully determined. It is obvious that differentiation with respect to $z$ gives functions with the desired properties on the surface of the cylinder, but odd derivatives must be excluded since they are not even in $z$ and cannot enter into the required solution. The function $\psi^{*}$ itself may also be included. The set of functions is therefore

$$
\psi^{*}, \quad \frac{\partial^{2} \psi^{*}}{\partial z^{2}}, \quad \frac{\partial^{4} \psi^{*}}{\partial z^{4}}, \cdots
$$

${ }^{2}$ See E. W. Hobson, Theory of spherical and ellipsoidal harmonics, Cambridge University Press, 1931, p. 94. Also cf. E. T. Whittaker and G. N. Watson, Modern analysis, Cambridge University Press, 1927, p. 325. The definition given in the latter is slightly different though it is also attributed to Hobson.

${ }^{3}$ See G. N. Watson, Theory of Bessel functions, 2nd edition, Cambridge University Press, 1944, p. 185. 
Since the use of any constant multiplier does not affect the desired properties, we may write the set of functions as follows:

$$
\psi_{2 s}^{*}=\frac{1}{(2 s-2) !} \frac{\partial^{2 s-2} \psi^{*}}{\partial z^{2 s-2}}
$$

the initial function being $\psi_{2}^{*}=\psi^{*}$.

To express the functions in terms of the spherical coordinates, the following relations are useful. ${ }^{4}$

$$
\frac{\partial^{2 s-2}}{\partial z^{2 s-2}}\left\{\frac{r^{2}}{\rho^{3}} P_{2}^{2}(\cos \phi)\right\}=3 r^{4} \frac{\partial^{2 s-2}}{\partial z^{2 s-2}}\left(\frac{1}{\rho^{5}}\right)=\frac{(2 s-2) ! r^{2}}{\rho^{2 s+1}} P_{2 s}^{2}(\cos \phi)
$$

and

$$
I_{2}(k r) \cos k z=-\sum_{n=1}^{\infty}(-1)^{n} \frac{(k \rho)^{2 n}}{(2 n+2) !} P_{2 n}^{2}(\cos \phi) .
$$

The latter expansion is valid in the neighborhood of the origin. Hence, it follows that for $s \geq 1$,

$$
\psi_{2 s}^{*}=r^{2} \frac{P_{2 s}^{2}(\cos \phi)}{\rho^{2 s+1}}-r^{2} \sum_{n=1}^{\infty}{ }^{2 n} \alpha_{2 s} P_{2 n}^{2}(\cos \phi) \rho^{2 n}
$$

where

$$
{ }^{2 n} \alpha_{2 s}=(-1)^{n+s}\left(\begin{array}{c}
2 n+2 s \\
2 s-2
\end{array}\right) \frac{\sigma_{2 n+2 s}}{2^{2 n+2 s}}
$$

in which, for $s \geq 4$,

$$
\sigma_{s}=\frac{2}{\pi} \frac{2^{s}}{s !} \int_{0}^{\infty} k^{s} \frac{K_{2}(k)}{I_{2}(k)} d k .
$$

Note that the asymptotic value of $\sigma_{s}$ is equal to unity.

By means of the above set of functions we construct

$$
\psi_{1}=\tau a \sum_{s=1}^{\infty} A_{2 s} \psi_{2 s}^{*},
$$

where $A_{2 s}$ are parametric coefficients to be determined; the factor $\tau a$ being introduced to render $A_{2 s}$ dimensionless. The function $\psi$ is then equal to

$$
\psi=\frac{\tau a r^{2}}{12} P_{2}^{2}(\cos \phi) \rho^{2}+\tau a r^{2} \sum_{n=1}^{\infty}\left(\frac{A_{2 n}}{\rho^{2 n+1}}-\rho^{2 n} \sum_{s=1}^{\infty}{ }^{2 n} \alpha_{2 s} A_{2 s}\right) P_{2 n}^{2}(\cos \phi) .
$$

Hence the boundary condition that $\psi=0$ when $\rho=\lambda$ is satisfied if, for $n \geq 1$,

$$
\frac{1}{12} \lambda^{2} \delta_{1, n}+\frac{A_{2 n}}{\lambda^{2 n+1}}-\lambda^{2 n} \sum_{s=1}^{\infty}{ }^{2 n} \alpha_{2 s} A_{2 s}=0
$$

or

$$
A_{2 n}=-\frac{1}{12} \lambda^{5} \delta_{1, n}+\lambda^{4 n+1} \sum_{s=1}^{\infty}{ }^{2 n} \alpha_{2 s} A_{2 s},
$$

where $\delta_{m, n}=1$ or 0 , according as $m=n$, or $m \neq n$.

${ }^{4}$ For the first relation see E. W. Hobson, Loc. cit., p. 105, formula 36. For the second relation, cf. T. M. MacRobert, Spherical harmonics, 2nd edition, Methuen, 1947, p. 362, Ex. 63. Also see the recurrence formulas for Legendre and Bessel functions. 
The above system of linear equations may be solved by successive approximations as follows. Write, for $n \geq 1$,

where

$$
A_{2 n}=\sum_{m=0}^{\infty} A_{2 n}^{(m)}
$$

$$
A_{2 n}^{(0)}=-\frac{1}{12} \lambda^{5} \delta_{1, n}, \quad A_{2 n}^{(m)}=\lambda^{4 n+1} \sum_{s=1}^{\infty}{ }^{2 n} \alpha_{2 s} A_{2 s}^{(m-1)}
$$

The validity of the above solution naturally depends upon the convergence of the series (32). To establish convergence, an inequality for the coefficients is needed. However, for the sake of brevity, no proof will be given here. From physical considerations alone, it seems likely that there will be convergence as long as the boundary of the cavity does not touch the surface of the cylinder or $\lambda<1$.

Effect of cavity on angle of twist. To investigate the effect of the cavity on the angle of twist, we have for $\psi_{0}$

$$
\frac{1}{a} \frac{\partial \omega_{0}}{\partial z}=\frac{1}{a r^{3}} \frac{\partial \psi_{0}}{\partial r}=\tau,
$$

where $\omega_{0}$ is the angle of twist due to $\psi_{0}$ alone. This implies that $\tau$ represents the angle of twist per unit length of a circular cylinder without a cavity. While for $\psi_{1}$, we have

$$
\frac{1}{a} \frac{\partial \omega_{1}}{\partial z}=\frac{1}{a r^{3}} \frac{\partial \psi_{1}}{\partial r}=\frac{\tau}{r^{3}} \sum_{s=1}^{\infty} A_{2 s} \frac{\partial \psi_{2 s}^{*}}{\partial r}
$$

where $\omega_{1}$ is the angle of twist due to $\psi_{1}$. Integration gives

$$
\omega_{1}=\frac{\tau a}{r^{3}} \sum_{s=1}^{\infty} A_{2 s} \frac{\partial}{\partial r} \int_{-\infty}^{\infty} \psi_{2 s}^{*} d z
$$

It appears that there is no contribution from $\psi_{2 s}^{*}$ except the integral part in $\psi_{2}^{*}$. The latter gives

$$
\begin{aligned}
\omega_{1} & =-\frac{4 \tau a A_{2}}{\pi r^{3}} \frac{\partial}{\partial r} r^{2} \int_{0}^{\infty} \int_{0}^{\infty} \frac{k^{2} K_{2}(k) I_{2}(k r)}{I_{2}(k)} \cos k z d z d k \\
& =-\frac{4 \tau a A_{2}}{\pi r} \operatorname{Lim}_{z \rightarrow \infty} \int_{0}^{\infty} \frac{k^{3} K_{2}(k) I_{1}(k r)}{I_{2}(k)} \frac{\sin k z}{k} d k \\
& =-\frac{2 \tau a A_{2}}{r} \operatorname{Lim}_{k \rightarrow 0}\left\{\frac{k^{3} K_{2}(k) I_{1}(k r)}{I_{2}(k)}\right\} \\
& =-16 \tau a A_{2}
\end{aligned}
$$

i.e., owing to the presence of the cavity the angle of twist of the cylinder is increased by an amount $-16 \tau a A_{2}$.

Numerical examples. Numerical examples will be given for several values of $\lambda$. Values of $\sigma_{s}$ are given in Table I, which are computed by numerical integration with the aid of Gregory's formula. ${ }^{5}$ The coefficients ${ }^{2 n} \alpha_{2 s}$ are then readily obtained and shown in Table II.

5See E. Whittaker and G. Robinson, Calculus of observations, 4th edition, Blackie 1948, p. 143. 
Values of $A_{2 s}$ found by successive approximations are shown in Table III. The stress at any point in the cylinder can now be readily computed. In particular, the shear stress across the minimum section $z=0$ is given by

$$
\tau_{\theta_{30}}=-\mu \tau a \sum_{n=1}^{\infty} A_{2 n}\left\{\frac{2 n-1}{r^{2 n+2}}+\frac{(2 n+2) r^{2 n-1}}{\lambda^{4 n+1}}\right\} P_{2 n}^{2}(0),
$$

where

$$
P_{2 n}^{2}(0)=(-1)^{n+1} \frac{(2 n+2) !}{2^{2 n}(n-1) !(n+1) !} .
$$

This stress is shown in Table IV and also graphically in Figure 1, where the known

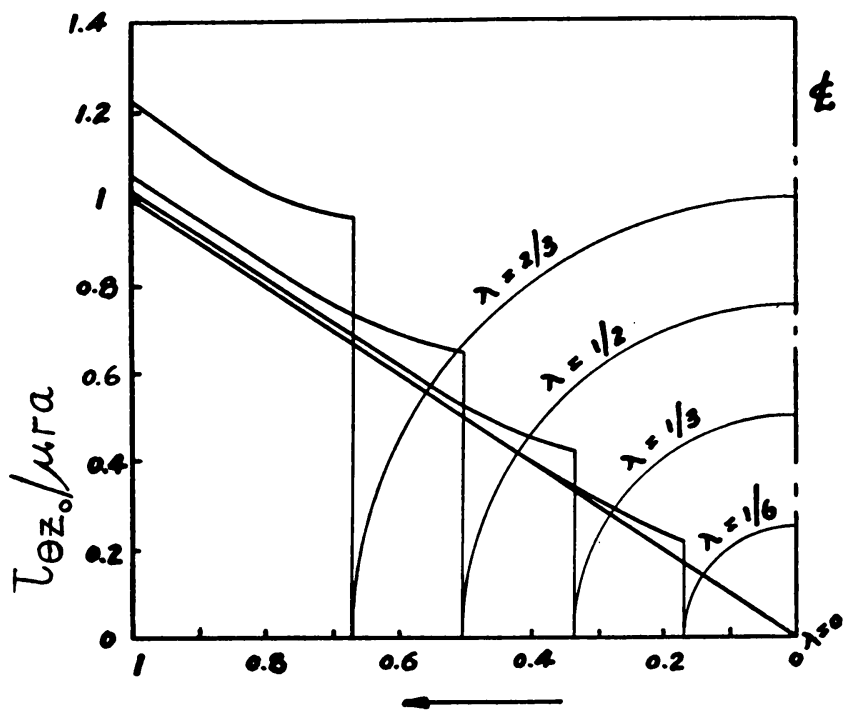

FIG. 1. Shear stress across minimum section

result for $\lambda=0$ is also included. It appears that in the cylinder under consideration the maximum shear stress always occurs on the external boundary across the minimum section. Now, if we define the stress concentration factor $K$ as the ratio of the maximum shear stress to the constant shear stress across the same section which would give the same couple about the axis of the cylinder, i.e.,

$$
K=\frac{4\left(1-\lambda^{3}\right)}{3 \mu \tau a}\left(\tau_{\theta_{z}}\right)_{\max }
$$

then the following results are obtained.

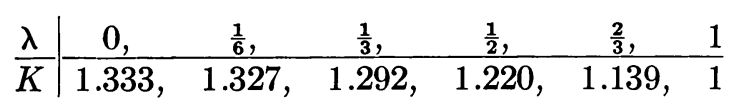

The value of $K$ in the limiting case $\lambda=1$ can readily be visualized from physical consideration of the cylinder. Figure 2 shows the results graphically. 


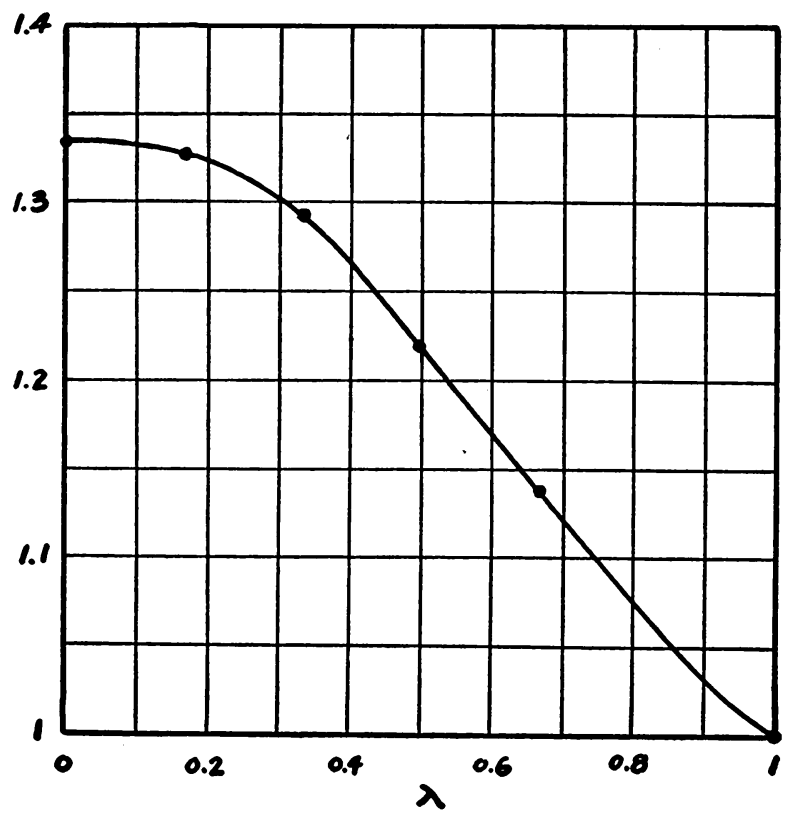

FIg. 2. The stress concentration factor $K$ versus $\lambda$.

TABle I. Coefficient $\sigma_{s}$

\begin{tabular}{c|cccccc}
\hline \hline$s$ & 4 & 6 & 8 & 10 & 12 \\
\hline$\sigma_{s}$ & 12.81758 & 4.15488 & 2.75876 & 2.20244 & 1.91108 & \\
\cline { 3 - 7 }$s$ & 14 & 16 & 18 & 20 & 22 & 24 \\
\hline$\sigma_{s}$ & 1.73288 & 1.61286 & 1.52653 & 1.46174 & 1.41078 & 1.37056 \\
\hline
\end{tabular}

TABLE II. Coefficient ${ }^{2 n} \alpha_{2 s}$

\begin{tabular}{r|r|r|r|r|r|r}
\hline \hline $2 s$ & \multicolumn{1}{c|}{$2 n=2$} & \multicolumn{1}{c|}{$2 n=4$} & $2 n=6$ & \multicolumn{1}{c}{$2 n=8$} & \multicolumn{1}{c}{$2 n=10$} & \multicolumn{1}{c}{$2 n=12$} \\
\hline 2 & $8.0110 \times 10^{-1}$ & $-6.4920 \times 10^{-2}$ & $1.0776 \times 10^{-2}$ & $-2.1508 \times 10^{-3}$ & $4.6657 \times 10^{-4}$ & $-1.0577 \times 10^{-4}$ \\
4 & $-9.7380 \times 10^{-1}$ & $2.9080 \times 10^{-1}$ & $-9.6787 \times 10^{-2}$ & $3.0794 \times 10^{-2}$ & $-9.6248 \times 10^{-3}$ & $2.9532 \times 10^{-3}$ \\
6 & $7.5435 \times 10^{-1}$ & $-4.5167 \times 10^{-1}$ & $2.3095 \times 10^{-1}$ & $-1.0587 \times 10^{-1}$ & $4.4791 \times 10^{-2}$ & $-1.7819 \times 10^{-2}$ \\
8 & $-4.5167 \times 10^{-1}$ & $4.3111 \times 10^{-1}$ & $-3.1762 \times 10^{-1}$ & $1.9708 \times 10^{-1}$ & $-1.0810 \times 10^{-1}$ & $5.4032 \times 10^{-2}$ \\
10 & $2.3095 \times 10^{-1}$ & $-3.1762 \times 10^{-1}$ & $3.1673 \times 10^{-1}$ & $-2.5481 \times 10^{-1}$ & $1.7561 \times 10^{-1}$ & $-1.0756 \times 10^{-1}$ \\
12 & $-1.0587 \times 10^{-1}$ & $1.9708 \times 10^{-1}$ & $-2.5481 \times 10^{-1}$ & $2.5755 \times 10^{-1}$ & $-2.1750 \times 10^{-1}$ & $1.6022 \times 10^{-1}$ \\
& & & & & \\
\hline
\end{tabular}


Table III. Coefficient $A_{2 n}$

\begin{tabular}{r|r|r|r|r}
\hline \hline $2 n$ & \multicolumn{1}{|c|}{$\lambda=\frac{1}{6}$} & \multicolumn{1}{c}{$\lambda=\frac{1}{3}$} & \multicolumn{1}{c}{$\lambda=\frac{1}{2}$} & \multicolumn{1}{c}{$\lambda=\frac{2}{3}$} \\
\cline { 2 - 4 } 2 & $-1.0718 \times 10^{-5}$ & $-3.4407 \times 10^{-4}$ & $-2.6710 \times 10^{-3}$ & $-1.2271 \times 10^{-2}$ \\
4 & $6.9045 \times 10^{-14}$ & $1.1348 \times 10^{-9}$ & $3.3887 \times 10^{-7}$ & $2.0889 \times 10^{-5}$ \\
6 & $-8.8434 \times 10^{-18}$ & $-2.3256 \times 10^{-12}$ & $-3.5176 \times 10^{-9}$ & $-6.9068 \times 10^{-7}$ \\
8 & $1.3619 \times 10^{-21}$ & $5.7307 \times 10^{-15}$ & $4.3912 \times 10^{-11}$ & $2.7521 \times 10^{-8}$ \\
10 & $-2.2796 \times 10^{-25}$ & $-1.5347 \times 10^{-17}$ & $-5.9586 \times 10^{-13}$ & $-1.1950 \times 10^{-9}$ \\
12 & $3.9873 \times 10^{-29}$ & $4.2955 \times 10^{-20}$ & $8.4513 \times 10^{-15}$ & $5.4393 \times 10^{-11}$ \\
& & & & \\
\hline
\end{tabular}

TABle IV. Shear Stress $\tau_{\theta z} / \mu \tau a$ Across $z=0$

\begin{tabular}{c|c|c|c|c|c}
\hline \hline$r$ & $\lambda=0$ & $\lambda=\frac{1}{6}$ & $\lambda=\frac{1}{3}$ & $\lambda=\frac{1}{2}$ & $\lambda=\frac{2}{3}$ \\
\hline$\lambda$ & 0.000 & 0.208 & 0.418 & 0.643 & 0.952 \\
$\frac{1}{4}+\frac{3}{4} \lambda$ & 0.250 & 0.377 & 0.518 & 0.696 & 0.977 \\
$\frac{1}{2}+\frac{1}{2} \lambda$ & 0.500 & 0.584 & 0.674 & 0.799 & 1.038 \\
$\frac{3}{4}+\frac{1}{4} \lambda$ & 0.750 & 0.792 & 0.858 & 0.919 & 1.119 \\
1 & 1.000 & 1.000 & 1.006 & 1.046 & 1.214 \\
\hline
\end{tabular}

